\title{
Transforming Care for Rare and Inherited Cardiovascular Diseases Through Education and Training
}

\author{
Perry M. Elliott \& Paolo Camici
}

The European Union defines a rare disease as a condition that affects less than 5 in 10,000 of the general population. By this definition, there are between 6,000 and 8,000 known rare disorders that together affect 1 in 17 Europeans. For this reason, rare disorders are the subject of intense scrutiny by governments and healthcare organisations that are developing new systems for clinical care, research and patient involvement.

There are many primary cardiovascular disorders including electrical, myocardial and vascular diseases that meet the current EU definition for rare conditions. In addition, a number of rare metabolic and neurological conditions are characterised by cardiovascular involvement that can be the major determinant of prognosis and quality of life. However, most cardiovascular services align predominantly to generic disease categories such as heart failure and coronary artery disease with little emphasis on the detection and treatment of less common conditions. As a result, many rare disorders are misdiagnosed or overlooked in everyday practice, resulting in suboptimal medical care and potentially adverse consequences for individuals and families.

The good news is that we live in a period of rapid medical innovation characterised by the emergence of new scientific disciplines such as genomics, proteomics and metabolomics. This new science offers great promise for the diagnosis of rare conditions and facilitates stratified (or personalised) medicine in which biological markers are used to separate patients into specific groups for treatment at a much earlier stage than is currently possible. The challenge for medical disciplines such as cardiology is to develop a workforce with the necessary skills to exploit these new scientific opportunities and thereby make a step change in healthcare for patients with uncommon cardiovascular disorders. 
In April 2017, some of the World's greatest experts in rare cardiovascular conditions came together in Athens, Greece to support the first International Course on Rare and Inherited Cardiovascular Disease supported by the Fondazione Internazionale Menarini [1]. The goal was to improve the education and training of cardiologists in the field of rare and genetic disorders and to prepare individual practitioners for the new era of personalized healthcare. With due deference to the host country, the course was 'Socratic' in style with an emphasis on interactive discussion between attendees and tutors and covered a broad range of topics including disorders of heart muscle, rhythm and large vessels.

In this special edition of The International Journal of Cardiology, several of the participating experts present short summaries of the course content that illustrate the core philosophy and aims of the meeting. Highlights include a pragmatic "rule out-rule in" algorithm designed to assist clinical diagnosis of unusual causes of common arrhythmia [2], new approaches to the complex pathophysiological architecture of dilated, hypertrophic and arrhythmogenic cardiomyopathies $[3,4,5]$, and a comprehensive overview of syndromic and non-syndromic diseases affecting large vessels [6]. The fundamental principle behind each article is that greater awareness of rare diseases coupled with the systematic application of readily available clinical tools provides the essential basis for accurate diagnosis and more effective therapy.

There are many factors that delay translation of new scientific discoveries into direct benefit for patients, but one of the most important is the failure to update and adapt clinical practice. Our sincere hope is that initiatives such as the Athens course will help to accelerate change by integrating the long-established methods of clinical diagnosis with the opportunities provided by molecular diagnosis and therapeutic innovation.

\section{References}

1. International Training Course on: Rare \& Inherited Cardiovascular Disease. http://www.en.fondazione-menarini.it/Home/News/European-Training-CourseonRare-Inherited-Cardiovascular-Disease-en-US 
2. Olivotto I, Finocchiaro G, Maurizi N, Crotti Lia. Common Presentation of Rare Cardiac Diseases: Arrhythmias. International Journal of Cardiology 2018;xxx

3. Linhart A, Cecchi F. Common presentation of rare diseases: Left ventricular hypertrophy and diastolic dysfunction. International Journal of Cardiology 2018;xxx

4. Anastasakis A, Basso C. Common presentation of rare diseases: The changing concept of dilated cardiomyopathy. International Journal of Cardiology 2018;xxx

5. Tsatsopoulou A, Bossone E. Common Presentation of Rare Diseases: Arrhythmogenic Right Ventricular Cardiomyopathy And Its Mimics. International Journal of Cardiology 2018;xxx

6. Arbustini E, Favalli V, Di Toro Alessandro, Giuliani L, Limongelli G. “Common presentation of rare diseases: aortic aneurysms \& valves". International Journal of Cardiology 2018;xxx 
Figure

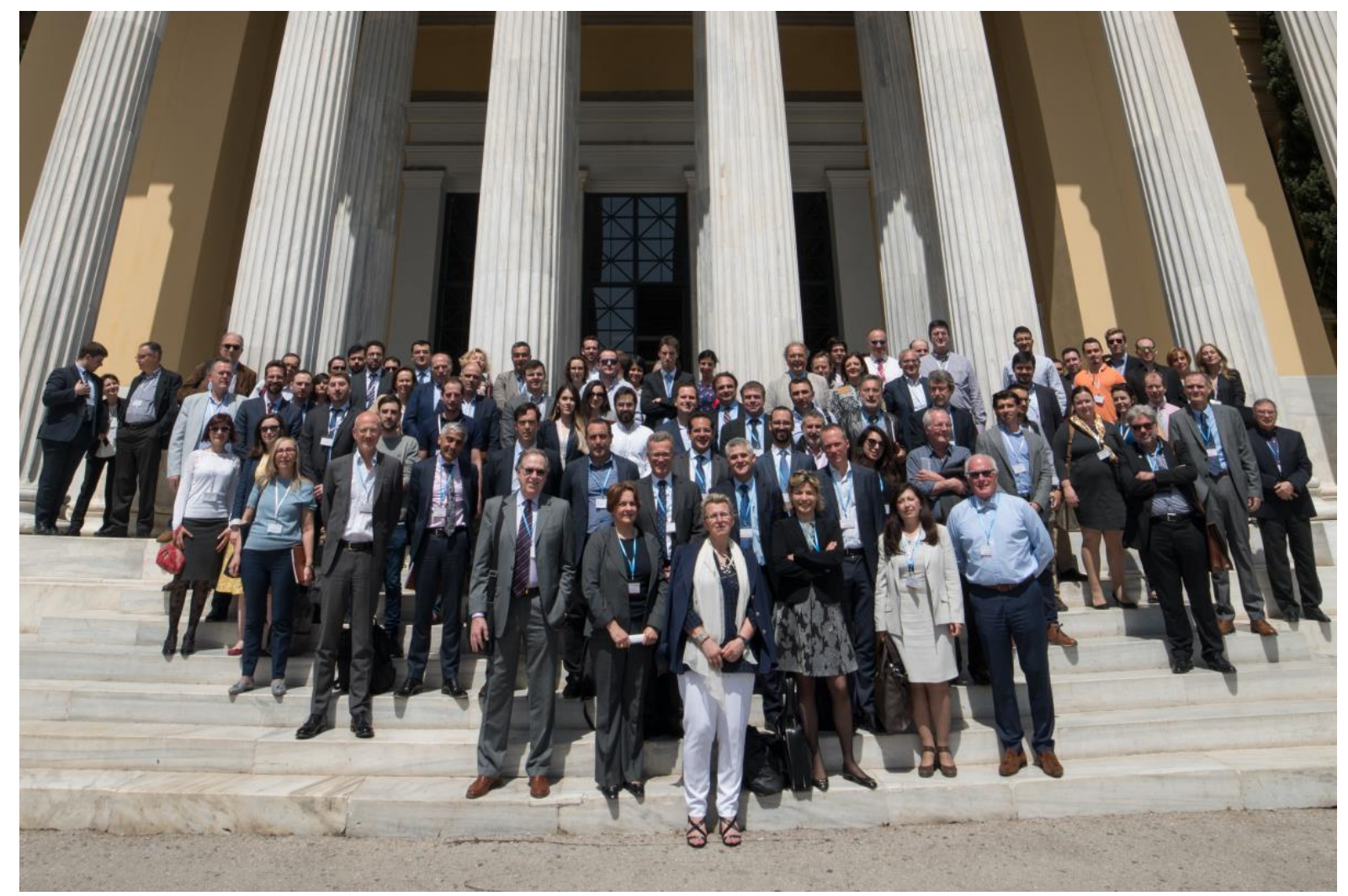

